\title{
Crisis Management on Residence: The Ten Elements of Effectiveness
}

\author{
Meg Driscoll \\ Former Manager Regional Accommodation \\ Accommodation Services \\ La Trobe University
}

\begin{abstract}
Given the likelihood of a negative incident occurring on campus, a simple but comprehensive framework was developed in 2016 at La Trobe University so that Accommodation Services staff could refer to a tool to assist them to respond to situations of crisis or potential crisis.

The Crisis Management Framework comprises ten elements. These ten elements are not sequential and are not to be interpreted as steps or stages. The central theme in this framework is that each element needs to be taken into consideration when responding to a crisis and delivered to varying degrees. The ten elements are:
\end{abstract}

- Co-ordinate, Control and Contain

- Cluster

- Communicate

- Create a place to Congregate and instil "Community"

- Collaborate

- Business Continuity

- Plan Comprehensively and Chronicle

- Care and Counsel

- Continuous Review and Improvement

- Celebrate

\section{Background}

On-campus accommodation is in many ways a microcosm of society and is therefore subject to the same potential for negative incidents as the broader population. Over the course of an academic year it is highly likely that at least one undesirable incident will occur within a residential precinct. University staff have duty-of-care obligations to provide the highest possible standard of health and safety and uphold their legislative obligations, ensure business continuity and support the university's reputation. It is imperative that managers and staff working within Accommodation Services and, to a lesser extent, the wider university's Emergency Management Team have an exemplary response to both sudden and smouldering crises within on-campus accommodation.

The ten elements of the Crisis Management Framework outlined in this paper were developed in 2016 by the Regional Manager of Accommodation Services at La Trobe University at that time, to ensure an effective response to the management of critical incidents on residence at La Trobe University. This comprehensive Framework may be useful for others working within a university context to follow, particularly those who work in on-campus accommodation and, specifically, the manager in charge. It is designed to support student residents and the broader community in relation to a range of incidents that may occur, with different levels of severity and for varying periods of duration.

The ten elements within this Framework are not intended to be sequential nor interpreted as steps or 
stages; they are simply a list of important controls for crisis co-ordinators who must respond effectively to a residential emergency. Each element needs to be considered in the context of the individual crisis and delivered to varying degrees throughout and after the crisis.

\section{Crisis management on residence: The ten elements of effectiveness}

\section{The crisis manager}

Effective crisis control within a university's residential context requires a Crisis Manager (CM) to oversee and take overarching responsibility for the incident at the residential level. The person with the highest authority who is working from the campus-in-crisis and within the Accommodation Service team would usually be the most appropriate person to take on this role. If the incident is small, the Manager in charge could take on this role. If the situation is larger or escalates, the role of CM should be nominated and/or endorsed by the university's Emergency Management Team. In these instances, it is advisable to also allocate an assistant to the CM. The assistant can manage record keeping, assist with the management of priorities and take on some of the CM's minor tasks so that the $\mathrm{CM}$ can meet the more important existing and additional obligations.

The CM is often required to make critical judgements during emergency situations under temporary or prolonged stress. An effective leader during a crisis will break each required action down into its component parts then allocate tasks, according to expertise, interest, and availability. When inspiration, expertise or support is needed, the CM must ask for it.

The following Crisis Management Framework is a list of the ten crucial components the Crisis Manager needs to consider when responding before, during and after an impending emergency within on-campus residential accommodation. 


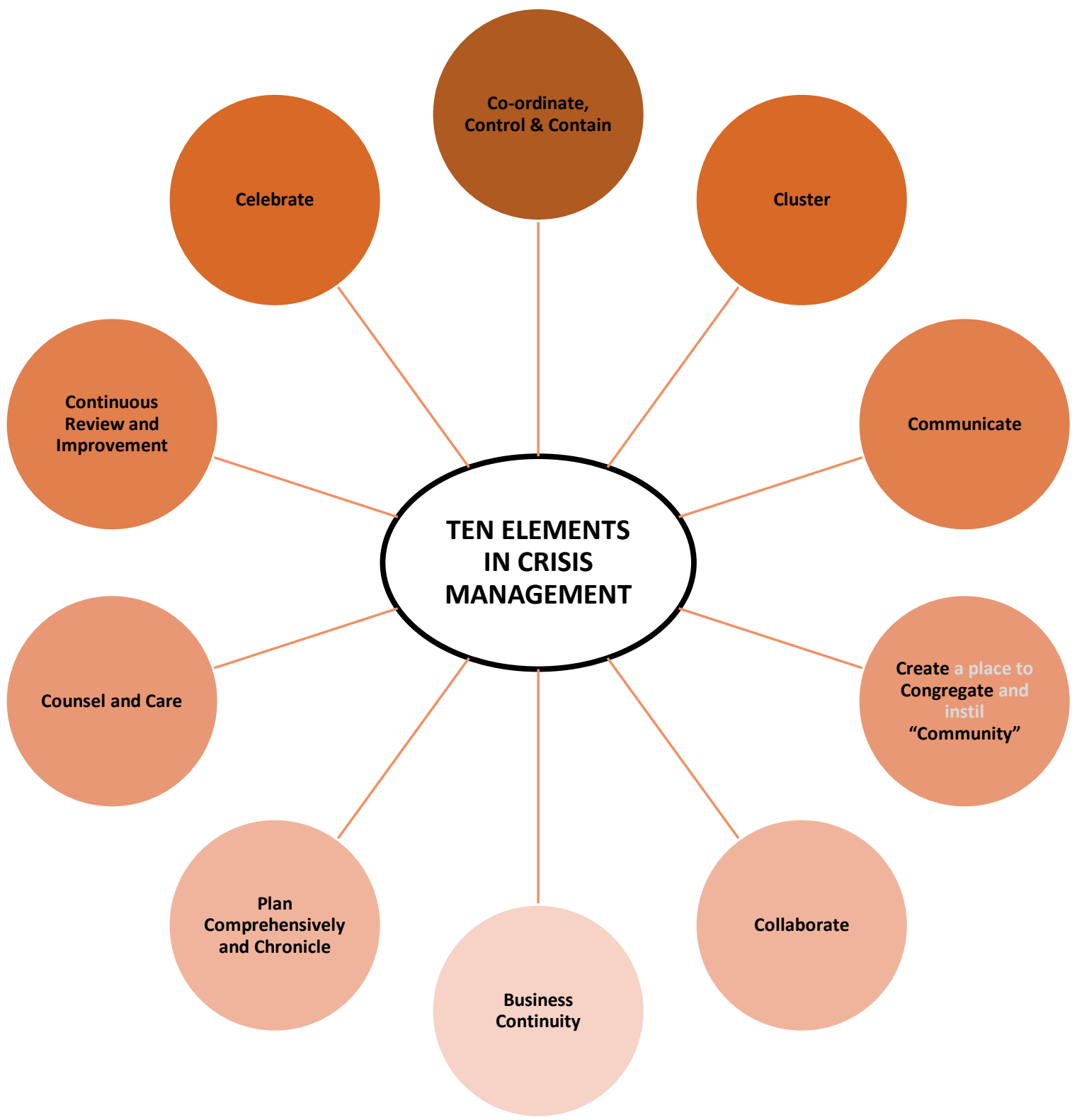

Figure 1: The Ten Elements in the Crisis Management Framework

\section{Element: Co-ordinate, Control and Contain}

One vital consideration for the Crisis Manager (CM) is to ensure effective co-ordination, control and containment of residents' verbal, emotional and physical responses to an incident so they can support positive outcomes and reduce negative consequences.

Firstly, the CM needs to ensure the location remains as unadulterated as possible as it might be a crime scene. Witnesses should be separated to ensure the integrity of their evidence and witness statements. Asking them to write down what happened whilst it is clear in their mind and until police arrive can be a beneficial approach.

Secondly, the CM needs to guide, influence, regulate or direct residents' communication (either verbal or electronic). The CM's leadership should include the establishment of a well-managed communication strategy that is clearly conveyed to staff and the residents. This strategy needs to regularly inform staff and residents, without sensation or unnecessary detail, what they should 
expect to see or hear. If residents are forewarned they are often more in control of their reactions and can practise their response in their mind.

The communication strategy also needs to take into consideration residents' interaction:

1. With other residents and students. Instruct them that they must not participate in unhelpful chit-chat, gossipy questions, rumours, misrepresentation or defamation associated with the incident and outline the consequences of such action. Provide residents with strategies and responses so they can shut things down confidently, politely, effectively and promptly;

2. With their families and friends external to the University. Encourage them to contact their loved ones and provide them with the message you would like them to send;

3. With the media. Give all students a standard response to use if they are approached by the media. Ask student leaders to inform their residents to forward all inquiries from the media to the $\mathrm{CM}$ and to inform the CM if the media approaches them; and

4. Via social media. Educate residents that social media is not always accurate, that their input can strongly influence the outcome of events, that what they put out there can be used as evidence or can make them liable to prosecution. Remind them that personal responsibility means managing impulses and encourage them to refrain from combing through information about a personally distressing matter.

Finally, the CM needs to contain residents' emotional responses to a crisis, some of which may risk a second incident emanating from the first. Sometimes anxiety, hysteria or anger can escalate an incident or lead to unmanageable behaviour. Empowering residents and organising meaningful ways they can assist with the crisis may reduce their stress, decrease their sense of helplessness, curb any vigilante response to the incident or prevent residents from taking any detrimental action (such as searching for a missing person themselves). Additionally, residents may need to be helped with ways to respond to a given situation.

\section{Element: Cluster}

There may be many people with whom the CM needs to communicate and the CM's messages often need to be tailored for different cohorts. The more intricate the crisis, the more important it is to organise cohorts into clusters. Cluster examples relevant to a crisis are "Team Members", "Student Leaders" and "Residents from the same Dorm"

The CM should obtain contact details of people within clusters and provide each cluster member with information about the cluster, its purpose, the people in the cluster and the type and frequency of communication they may receive. The invitation may not be accepted and people may suggest others to be part of a cluster. The CM should then liaise with the cluster members to determine the most effective communication channel for each cluster and also, when numbers are large, establish one person to be the representative of that cluster and through whom communication is channelled. Throughout the crisis, the CM should keep updated details pertaining to each cluster, contact details of those within each cluster and the communication they have provided and received. An example of the flow of communication from the CM can be found in Figure 2.

\section{Element: Communicate}

The CM must ensure all communication is done regularly and with clarity, professionalism and integrity. This practice provides residents and staff with reassurance, prevents anxiety and panic 
and ensures the truth is being circulated.

A CM should assure residents and staff that accurate information will be forwarded to them and inform them of its medium and frequency, taking into consideration Privacy Laws and the restrictions associated with crime investigations. When the crisis is at its peak, information may be as frequent as every hour, even if it is informing stakeholders that there are no updates. Whilst emails and texts can be quick and efficient, the CM should deliver some face-to-face updates in order to observe people, look for signs of stress or exhaustion and follow up accordingly.

Staff who have been tasked with keeping business continuity so that others can be freed to take on additional duties and respond directly to the crisis should be treated as part of the response team and given regular updates on the situation.

As shown in the Flowchart of Communication (Figure 2), all information pertaining to the crisis is to be channelled through the $\mathrm{CM}$ who will pass on to individuals, clusters, colleagues and/or external bodies such as the police, accordingly. This clear line of communication should be established and shared with all stakeholders. It should also include informing people when there is a change of command. Through this process, the CM can keep abreast of the facts and falsehoods circulating in the broader community, check for accuracy of information and correct any untruths.

At the onset of a crisis, a dedicated CM's mobile phone number and email address need to be established and communicated to all stakeholders. The $\mathrm{CM}$ can redirect unrelated inquiries to another line and hand over the mobile phone and a dedicated notebook to a relieving $\mathrm{CM}$ if the crisis lasts longer than ten hours.

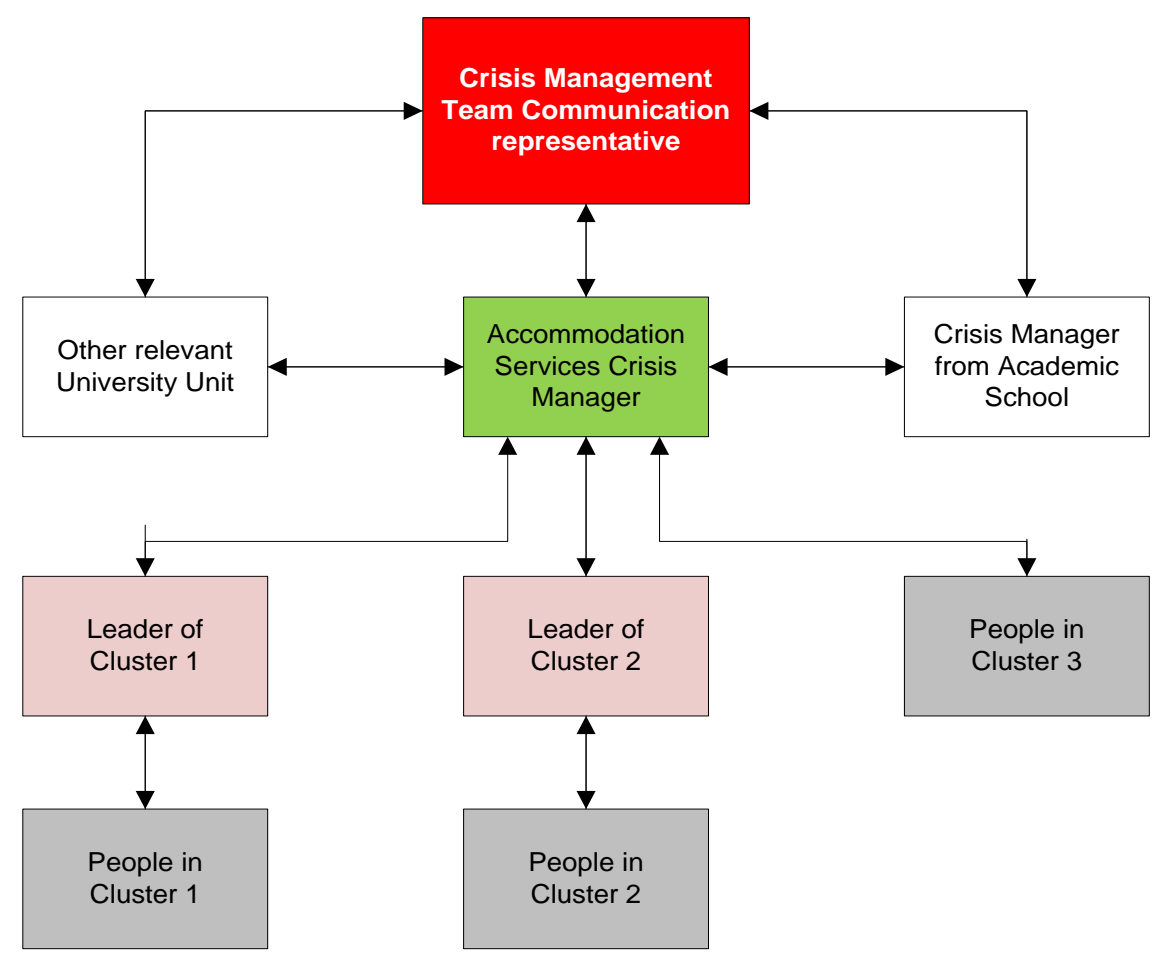

Figure 2: The Crisis Management Framework Flowchart of Communication

\section{Element: Create a place to Congregate and instil "Community"}

Especially during a crisis, residents should be encouraged to support each other and look out for each other in their on-campus home. Dispatches and conversations should include messages about 
sharing, respect, unity and empathy.

Depending on the crisis, the CM may need to create and convey to all residents the availability of a "safe" community place where they can congregate any time during and, if necessary, after the crisis. It should be a comfortable and calming sanctuary, conveniently located on campus for easy access and a staff member should be stationed there to provide support. Residents should be encouraged to go there if they are distressed. This place should be the meeting place for any activity or community announcement relating to the incident. If possible, there should be a kitchenette to ensure drinks and healthy food can be provided and a separate meeting room.

\section{Element: Collaborate}

A team approach across university divisions is essential during a crisis. Most universities have a Crisis Management Team (CMT) with overarching responsibility for all emergency situations. The $\mathrm{CM}$ will be managing the demands of the crisis at the residential level but must act as directed by the CMT, to maintain constant communication and take the time to attend all CMT meetings and updates. Other important collaborative arrangements to consider are:

- The CM's direct supervisor

- Academic Schools - staff need to know who may be affected by the crisis so they have empathy for anxious students or student absences and can consider reasonable adjustments during and after the crisis,

- The Media Relations Unit (MRU)- approval for major communication announcements needs to be obtained and the MRU needs to be abreast of the situation,

- The HR Division and the Occupational Health \& Safety (OHS)Team - these specialists can provide advice and ensure decisions made in relation to the CM's control are within regulations,

- Support Teams such as cleaners, security staff, chaplains and counsellors and event managers who will need to know if there might be an increase or change to the demands of their job or may need to change their own plans and events as a result of the situation.

\section{Element: Business Continuity}

The CM is required to have a balanced approach to two important priorities - the crisis and business continuity. The reputation of the business cannot suffer. Residents pay rent and fees, they expect a high level of professional service at all times. Crisis or not, phones ring, future students and their families require attention, students with health and wellbeing issues need support, the business must collect rent and follow up overdue payments, planned social and academic programs and events need to be delivered or, if this is not appropriate, they must be postponed or cancelled.

\section{Element: Plan Comprehensively and Chronicle}

The CM must commence comprehensive planning from the moment a potential crisis emerges, regularly referring to the Ten Elements of Effective Management and chronicling everything with precision and detail.

The CM has the responsibility for the completion of a working blueprint containing comprehensive plans and a chronicle of all matters pertaining to the crisis and business continuity. If the crisis 
persists for more than 24 hours, a longer-term plan is required; if it is complex, planning may need to include a brainstorm of different crisis scenarios and possible tasks and resources for each. When the $\mathrm{CM}$ is relieved, the relieving $\mathrm{CM}$ takes on the responsibility for comprehensively planning and chronicling the crisis. Chronicling should be in written form, but the CM can make notes via notebook, digital recording or even using a whiteboard marker on glass to record as necessary, and then transfer information to the blueprint when there is more time.

The blueprint will include a daily program which includes recording meetings, their attendees and outcomes, plus communication schedules, staff rosters and events. No team member, including the $\mathrm{CM}$, should work longer than five hours without a rest and sustenance, while staff schedules should ensure every team member has at least 8 in 24 hours away from responsibility.

All required tasks will be listed, as well as the personnel and resources required to complete them. A tally of costs will be kept and all agreements and assurances will be documented. Lists of key stakeholders and clusters and their contact details will be added, as will ingoing and outgoing communiques (phone calls, texts and emails). The CM will include personal notations regularly with arising issues, actions and outcomes. The blueprint will be amended with additional facts, including any changes, cessation or additions to plans. There will be a chronicle of pre-, mid- and post- crisis action, of celebration and contributions. It is critically important that timetabling and planning includes time-out for staff rest.

Planning and chronicling is vital for business continuity, effective crisis management, continuous improvement and post-crisis review. Documentation may also assist the $\mathrm{CM}$ when required to recollect facts, provide evidence in a court of law or demonstrate duty-of-care and professionalism.

\section{Element: Care and Counsel}

A proactive response to the health and wellbeing of staff, residents, student leaders and support personnel is required during a crisis; stress and harm need to be minimised, emotional support must be provided, and individuals should be given coping strategies. From the outset, the CM needs to organise for intervention for individuals so they can deal with their response to the situation, acknowledging that the impact of a crisis often goes beyond those directly involved in an incident. This can include engaging educators and mental health practitioners and ensuring relevant personnel have sufficient training to effectively offer unconditional acceptance, reassurance and support. Care also includes supplying a safe place and somebody to talk to, the provision of healthy food and drink, assistance with assignment extensions, opportunities for relaxation and positive thinking, and access to resources and lists of support networks, thus enabling individuals who choose to take personal responsibility for their wellbeing, to do so in a private way.

\section{Element: Continuous Review, Improvement}

A process of continuous review and improvement should take place during a crisis at the end of each day or at a particular milestone. The format of the review is dependent upon the matter being assessed and can range from a formal team meeting to an informal brainstorm undertaken done by the CM.

After the crisis (exactly when, depends on the severity of the incident) the CM should call a meeting and invite team members, superiors, representatives from clusters and/or members from the university's OHS Team, or Emergency Response Team, and conduct a formal analysis of the processes and actions undertaken before, during and after the crisis. A Crisis Report should ensue, with minutes from the meeting and a list of good practice, oversights, failures, lessons learned and 
acknowledgements. It should contain a list of action items emanating from the review, those responsible for them and dates by which they should be completed. A person should be put in charge to ensure these actions are undertaken and the Crisis Report should be updated accordingly.

\section{Element: Celebrate}

Celebrate the good things about the outcomes and the teamwork achieved. Appreciate the work of others and take time to say "thanks" for specific contributions, however small,and the achievement of major milestones during and after a crisis.

\section{Conclusion}

Residential precincts are not exempt from crises and, despite efficient preventative actions and processes, some emergencies just cannot be avoided. Accommodation Services' personnel, particularly a nominated Crisis Manager, need operational tools to assist them as they strive to achieve outcomes that are as safe, swift and successful as possible. These tools need to include crisis planning and reporting solutions, provide strategies for managing and communicating with all stakeholders and ensuring their health and wellbeing, and prompt Crisis Managers to undertake continuous review and show appreciation for the efforts of others.

Support staff working with students who reside on campus can use this Framework of Ten Elements of Effectiveness as a reference point to prevent overlooking important aspects of crisis management on residence.

The author may be contacted:

meg.driscoll@outlook.com

\section{Please cite this paper as:}

Driscoll, M. (2018) Crisis management on residence: The ten elements of effectiveness. Journal of the Australian and New Zealand Student Services Association. 26(2), 121-128. https://doi.org.10.30688/janzssa.2018.12 